\title{
Near-field 2-D and 3-D Radar Imaging Using a Chirp Scaling Algorithm
}

\author{
Encarna Gimeno and Juan M. Lopez-Sanchez
}

\author{
Departamento de Física, Ing. Sistemas y Teoría de la Señal (DFISTS), University of Alicante \\ P.O. Box 99, E-03080 Alicante, Spain \\ Phone / Fax: 34965909597 / 3464, E-mail: encarna@disc.ua.es E-mail:juanma@disc.ua.es
}

\begin{abstract}
A new chirp scaling algorithm (CSA) has been formulated and tested. This method yields more accurate results when applied to configurations with high coherent integration angles and high bandwidth-to-center-frequency ratios than the original CSA. Moreover, it has been extended to the three-dimensional case when a planar synthetic aperture is employed. The formulation of the new method is justified in this work. In addition, an algorithm version adapted to stepped-frequency radars has been developed. Finally, this technique is implemented and successfully tested with numerical simulations and real data obtained with a ground-based system.
\end{abstract}

\section{INTRODUCTION}

The task of any synthetic aperture radar (SAR) processor is to focus the signal data in both range and azimuth directions. Processing problems are range curvature or range cell migration, and that this range cell migration depends on range. Moreover, the design of a SAR processor is harder under the following conditions: 1) high coherent integration angles (ratio of the synthetic aperture length over the distance from antenna to scene center), and 2) high bandwidth-to-center-frequency ratios, $B / f_{c}$. We refer to these extreme conditions as near-field conditions. This situation is common when working in highresolution radar configurations with ground-based systems and anechoic chambers.

The range migration algorithm (RMA), or $\omega-k$, which works in the range-frequency/azimuth-frequency domain, achieves an excellent accuracy in near-field conditions [1]. But this algorithm includes an interpolation to perform the range migration correction. An alternative method to avoid this computationally demanding step is the chirp scaling algorithm (CSA) [2]. This technique makes use of the linear FM (chirp) nature of the transmitted pulse in order to scale the range time axis in the range/azimuth-frequency domain. Its implementation requires only FFT's and complex products. In the original CSA, the phase functions that are used to focus the data only yield approximate results and do not produce accurate images in nearfield cases.

The contribution of the present work is the extension of the efficient CSA to the near-field case when applied in two and three-dimensional images. The original 2-D CSA [2] is reformulated in order to incorporate additional terms in the approximations assumed by the algorithm. These terms are required to maintain the accuracy of the method in near-field conditions, but do not entail a significant increase in the computation time. In addition, a new 3-D CSA method is formulated by extending the original method (with a linear aperture) to a general case with a planar synthetic aperture. The new formulation enables the generation of 3-D images with excellent radiometric and geometric accuracy. The method is also optimized for stepped-frequency radars.

The performance of the method has been illustrated with numerical simulations and experiments. Experimental results have been obtained by employing radar data from the European Microwave Signature Laboratory (EMSL) and the outdoor linear SAR system (LISA), both at JRC Ispra, Italy.

\section{FORMULATION OF THE NEAR-FIELD CSA}

The chirp scaling algorithm operates in both rangefrequency/azimuth-frequency domain and range/azimuthfrequency domain, and it requires range-chirped signal data. Extending the formulation of the original CSA for a linear aperture [2, 3], we have obtained similar expressions for a 3-D algorithm with a planar aperture. The 2-D CSA results a particular case of 3-D CSA when the aperture size in one dimension (say $z$ ) is zero. Fig. 1 shows the block diagram of the new 3-D CSA.

The first step of the original CSA formulation for a linear aperture [3] is an azimuth Fourier transform of the SAR signal data from a point scatterer. The expression of this transform can be approximated by the method of stationary phase (MSP) in a four-stage procedure. The procedure begins with the range Fourier transform of the signal impulse response (first application of the MSP), followed by an azimuth Fourier transform of the resulting expression (again by MSP), and a simplifying approximation of Taylor's series expansion of its phase. In the range-frequency/azimuth-frequency domain, the 3-D point scatterer response phase expressed as a series in $f_{\tau}$ is,

$$
\Phi\left(f_{\tau}, f_{x}, f_{z} ; r\right)=\sum_{k=0}^{\infty} \phi_{k}\left(f_{x}, f_{z} ; r\right) f_{\tau}^{k}
$$

This expression makes possible to control the approximation error for near-field by a phase function multiplier in the rangefrequency/azimuth-frequency domain. The original 2-D CSA only includes up to the second order of this expansion. This approximation enables the determination of the inverse Fourier 
transform in the range dimension (by MSP) to obtain the desired azimuth Fourier transform of the original expression. The key point of the new near-field algorithm consists in transforming the chirped SAR data into the range-frequency/azimuthfrequency domain and adding a multiplier phase function that compensates the rest of required terms in the Taylor's expansion at a reference range. The highest term in this phase function is determined by the image accuracy required and the importance of the near-field situation. The rest of stages are equivalent to the original CSA. The phase function of $n$-th order can be expressed as,

$$
S_{r e f 0}\left(f_{\tau}, f_{x}, f_{z} ; r\right)=\sum_{k=3}^{n} \exp \left\{-j \phi_{k}\left(f_{x}, f_{z} ; r\right) f_{\tau}^{k}\right\}
$$

where

$$
\begin{aligned}
& \phi_{k}\left(f_{x}, f_{z} ; r\right)= \\
& =\frac{1}{2^{k}} \frac{4 \pi \lambda^{k-1}}{c^{k}} r \sum_{j=\operatorname{ceiling}\left(\frac{k}{2}\right)}^{k} \frac{(-1)^{j-1} 2^{j} \cdot \Pi_{i=1}^{j-1}(2 i-1)}{A_{x z}^{2 j-1} \cdot(2 j-k) !(k-j) !}
\end{aligned}
$$

$c$ is the speed of light, $\lambda$ is the radar central wavelength, and

$$
A_{x z}\left(f_{x}, f_{z}\right)=\sqrt{1-\left(\frac{\lambda f_{x}}{2}\right)^{2}-\left(\frac{\lambda f_{z}}{2}\right)^{2}} .
$$

The near-field CSA method can be easily adapted to SAR data obtained by stepped-frequency radars. With this type of radar, data are not chirped, so we have to include a phase term to rechirp the data. Since the stepped-frequency radar data are in the range-frequency/azimuth domain, they can be transformed by a simple FFT to the range-frequency/azimuthfrequency domain, and then perform the required near-field approximation. Fig. 2 shows the block diagram of the new 3-D CSA.

\section{RESULTS}

The performance of the algorithm has been tested with numerical simulations and real data sets obtained using an outdoor linear SAR system. The code of the algorithm has been implemented in $C$ programming language. Due to space constraint, only one numerical simulation is shown here.

The target used in this numerical simulation consists of a 3-D array of $5 \times 5 \times 5$ point scatterers uniformly distributed within a box of side $1 \mathrm{~m}$. All scatterers have the same RCS: $0 \mathrm{dBsm}$. A TX/RX antenna synthesizes a planar aperture of $2 \mathrm{~m} \times 2 \mathrm{~m}$ located at $r_{o}=2 \mathrm{~m}$ from the target center. The number of measurement points is 101 , spaced $2 \mathrm{~cm}$, both in the horizontal and vertical cross-range directions. These sampling intervals have been selected according to the Nyquist criterion. The frequency ranges from 2 to $6 \mathrm{GHz}$, sampling a total of 169 points with a step of about $24 \mathrm{MHz}$. According to these parameters the theoretical resolution is $3.75 \mathrm{~cm}$ along the three main axes. Note

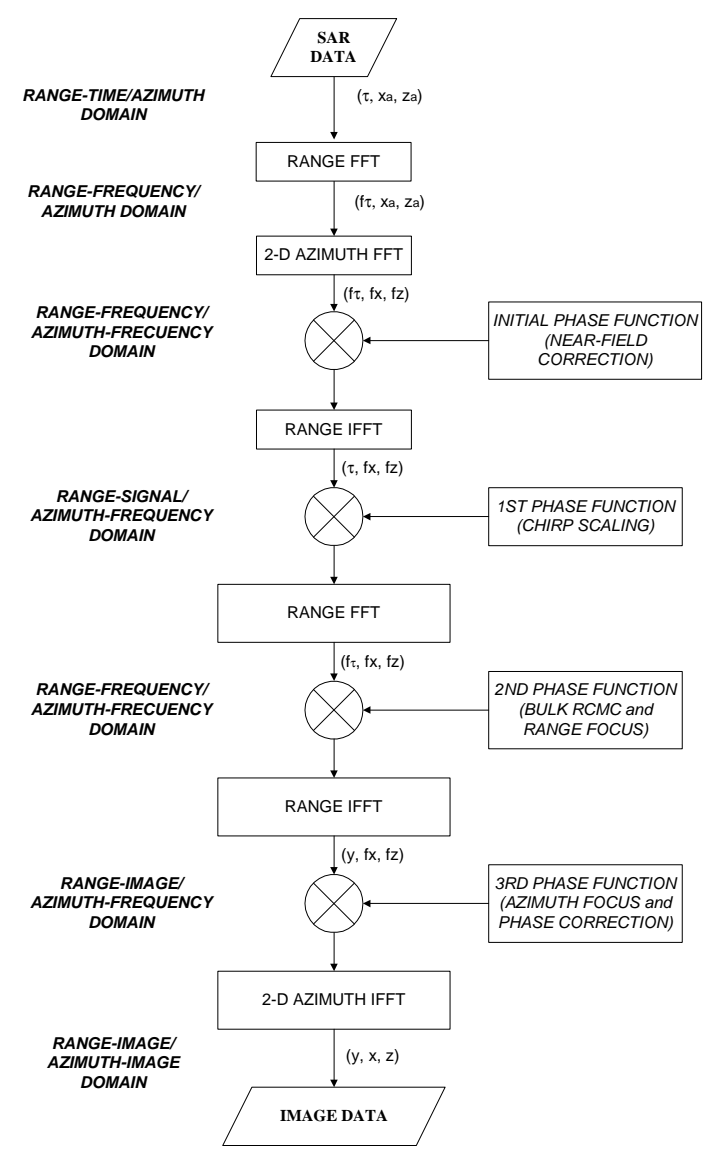

Fig. 1. Flow diagram of the near field 3-D CSA for chirped data.

that this set-up presents strong processing requirements, since both integration angle and bandwidth-to-center-frequency ratios are very high.

Fig. 3 and 4 shows the slices of the image onto an azimuth and the range planes, respectively. The quality of the reconstructed image is quite satisfactory and, moreover, the processing is about 50 percent shorter than the recently published 3-D RMA in the same simulation [1].

Additional simulations have shown the dynamic range that can be achieved depending on the measurement configuration. In all cases it is better than $70 \mathrm{~dB}$. Other quality parameters, like the impulse response have been analyzed in this work (Fig. 5 and 6).

\section{CONCLUSiONS}

The original 2-D CSA has been extended to the 3-D case and some additional terms have been formulated and included to obtain accurate images in near-field configurations. This method has been tested with simulations and experiments. More details will be presented at the time of the conference.

The extension of the method to cylindrical and spherical scanning geometries, common in anechoic chambers, is sub- 


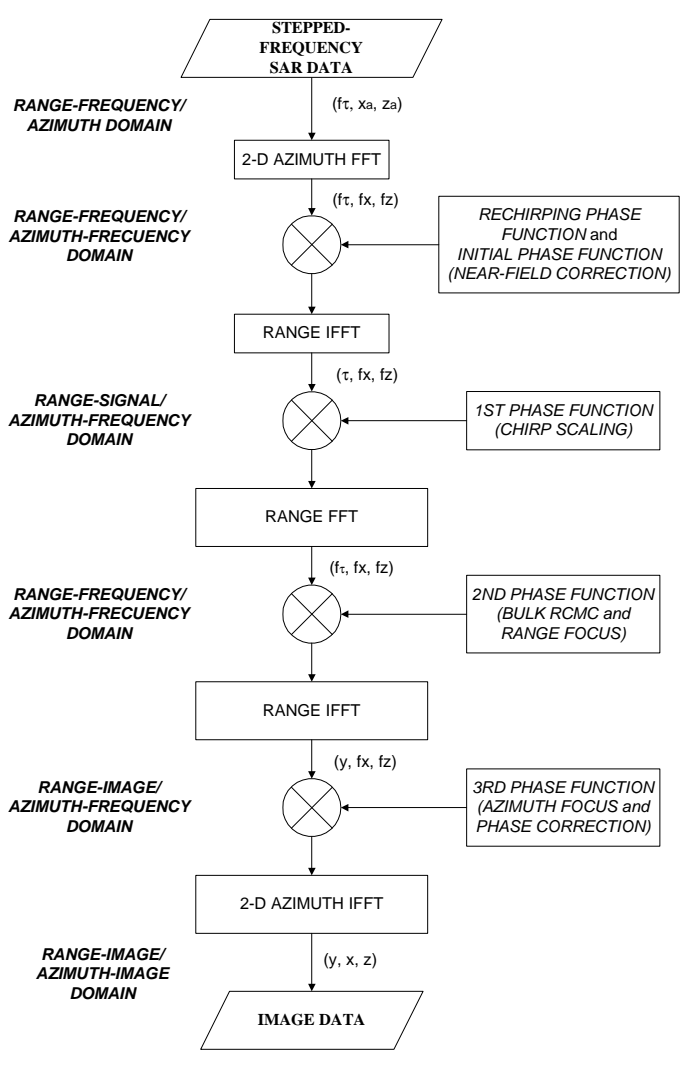

Fig. 2. Flow diagram of the near field 3-D CSA for stepped-frequency radar data.

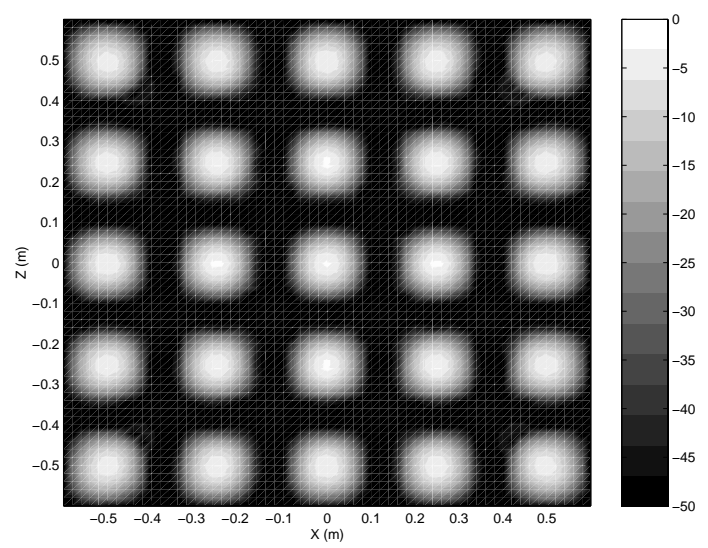

Fig. 3. Slice of the 3-D reflectivity image at constant range $y=0$.

ject of an ongoing research.

\section{REFERENCES}

[1] J. M. Lopez-Sanchez and J. Fortuny, "3-D radar imaging using range migration techniques," IEEE Trans. Antennas Propagat., vol. 48, pp. 728737, May 2000.

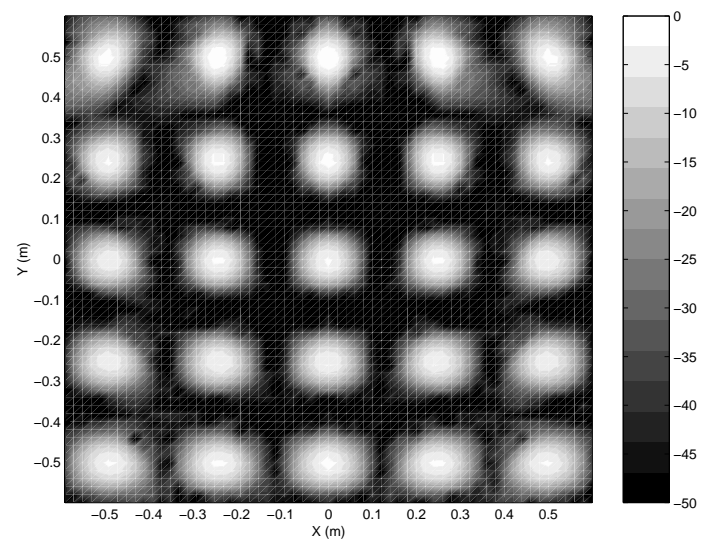

Fig. 4. Slice of the 3-D reflectivity image at constant azimuth $z=0$.



Fig. 5. 2-D point scatterer response of SAR system at constant range $y=0$.

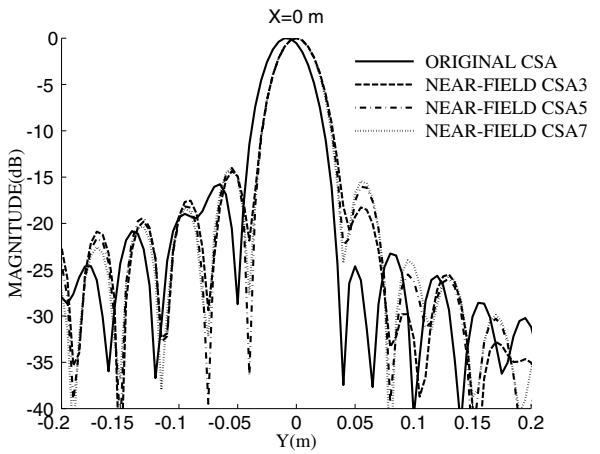

Fig. 6. 2-D point scatterer response of SAR system at constant azimuth $x=0$.

[2] R. K. Raney, H. Runge, R. Bamler, I. G. Cumming, and F. H. Wong, "Precision SAR processing using chirp scaling," IEEE Trans. Geosci. Remote Sensing, vol. 32, pp. 786-799, July 1994.

[3] W. G. Carrara, R. S. Goodman, and R. M. Majewski, Spotlight Synthetic Aperture Radar. Signal Processing Algorithms. Artech House, 1995.

\section{ACKNOWLEDGMENT}

This work is partially supported by the Generalitat Valenciana under Project No. GV00-036-14. 